\title{
Correction to: Effect of Nb Content on Microstructures and Mechanical Properties of Ti-xNb-2Fe Alloys
}

Qiang Li (D), Pu Miao, Junjie Li, Meifeng He, Masaaki Nakai, Mitsuo Niinomi, Akihiko Chiba, Takayoshi Nakano, Xuyan Liu, Kai Zhou, and Deng Pan

\section{Correction to: JMEPEG https://doi.org/10.1007/s11665-019-04250-5}

There was an error in the text of this article as originally published.
In the Microstructure section of the Results, " $332_{\beta}\langle 113\rangle_{\beta}$ " should be " $\{332\}_{\beta}\langle 113\rangle_{\beta}$ " (in two places) because " $\{332\}_{\beta}$ $\langle 113\rangle_{\beta}$ " is the expression of twinning.

The original version of the article was revised.

Publisher's Note Springer Nature remains neutral with regard to jurisdictional claims in published maps and institutional affiliations.
The original article can be found online at https://doi.org/10.1007/ s11665-019-04250-5.

Qiang Li, Pu Miao, Xuyan Liu, and Kai Zhou, School of Mechanical Engineering, University of Shanghai for Science and Technology, Shanghai 200093, People's Republic of China; Junjie Li, CAS Key Laboratory of Functional Materials and Devices for Special Environments, Xinjiang Technical Institute of Physics and Chemistry, CAS, Xinjiang Key Laboratory of Electronic Information Materials and Devices, 40-1 South Beijing Road, Ürümqi 830011, China; Meifeng He, School of Materials Science and Engineering, University of Shanghai for Science and Technology, Shanghai 200093, People's Republic of China; Masaaki Nakai, Department of Mechanical Engineering, Faculty of Science and Engineering, Kindai University, 3-4-1 Kowakae, Higashiōsaka, Osaka 577-8502, Japan; Mitsuo Niinomi, School of Mechanical Engineering, University of Shanghai for Science and Technology, Shanghai 200093, People's Republic of China; Institute for Materials Research, Tohoku University, 2-1-1, Katahira, Aoba-ku, Sendai 980-5377, Japan; Department of Materials and Manufacturing Science, Graduate School of Engineering, Osaka University, 2-1, Yamada-Oka, Suita, Osaka 565-0871, Japan; Department of Materials Science and Engineering, Graduate School of Science and Technology, Meijo University, 1-501, Shiogamaguchi, Tempaku-ku, Nagoya 468-8502, Japan; Institute of Materials and Systems for Sustainability, Nagoya University, Furo-cho, Chikusa-ku, Nagoya 464-8601, Japan; and Faculty of Chemistry, Materials and Bioengineering, Kansai University, Osaka 564-860, Japan; Akihiko Chiba, Institute for Materials Research, Tohoku University, 2-1-1, Katahira, Aoba-ku, Sendai 980-5377, Japan; Takayoshi Nakano, Department of Materials and Manufacturing Science, Graduate School of Engineering, Osaka University, 2-1, Yamada-Oka, Suita, Osaka 565-0871, Japan; and Deng Pan, Materials Genome Institute, Shanghai University, Shanghai 200444, People's Republic of China. Contact e-mails: jxli@tju.edu.cn and niinomi@imr.tohoku.ac.jp. 